\title{
Outcome of SARS-CoV-2 in pregnancy and newborns - is there a new kid in the TORCH block?
}

\author{
Andreia Florina Nita', Sabina Cornelia Manolescu', \\ Mircea Ioan Popa ${ }^{1,2}$, Loredana Gabriela Popa ${ }^{1,3}$ \\ ${ }^{1 " C a r o l ~ D a v i l a " ~ U n i v e r s i t y ~ o f ~ M e d i c i n e ~ a n d ~ P h a r m a c y, ~ B u c h a r e s t, ~ R o m a n i a ~}$ \\ 2"Cantacuzino" National Medico-Military Institute for Research and Development, \\ Bucharest, Romania \\ ${ }^{3}$ Colentina Clinical Hospital, Bucharest, Romania
}

\begin{abstract}
Objective. This study aimed to perform a systematic review of existing literature to assess the outcomes of pregnancy in women with COVID-19 infection and their newborns while estimating the possibility of vertical transmission.

Materials and methods. We conducted a systematic literature research using Pubmed and Google Scholar covering the period from December 2019 to 20th of November 2020. The review was conducted in accordance with PRISMA guidelines.

Outcomes. We included 16 studies - systematic reviews and meta-analyses published between May 2020 and November 2020 - which focused on perinatal outcomes of pregnant women with COVID-19 and 7 case reports of neonates with congenital transmission of COVID-19. Overall, the rate of COVID-19 cases in neonates of COVID-19 positive mothers was $3 \%$ with $95 \% \mathrm{Cl}[1.86,4.24])$. The preterm birth rate was $16.4 \%$ with $95 \% \mathrm{Cl}[10.5,22.3]$ and the rate of stillbirths and foetal deaths was $1.4 \%$ (11 studies, 0 to $4.8 \%$ ). From the 7 newborns with proved vertical transmission, majority were born preterm, with good birth weight and APGAR score and heterogenous symptoms; 4 developed severe symptoms. Overall progress and evolution for both mother and newborn was good.

Conclusions. COVID-19 impact on pregnancy outcome is similar to general population in regard to preterm rate and stillbirth rate. Vertical transmission is possible and it seems to occur in about $3 \%$ of cases. Overall maternal and perinatal outcome is favourable and clinical presentation of in utero transmission of SARSCoV-2 in newborns is heterogenous.
\end{abstract}

Keywords: COVID-19, vertical transmission, neonates, congenital infection

\section{INTRODUCTION}

On $11^{\text {th }}$ of March 2020 the new coronavirus infection was declared a global pandemic by World Health Organization (WHO). Cumulatively as of 15 November 2020, 53.7 million confirmed cases and 1.3 million deaths have been reported to WHO (1).

Worldwide, there are about 210 million pregnancies annually with an estimated number of 170 million births (2).
It is well known that the physiological changes that occur during pregnancy make the woman vulnerable to severe infections and the pregnant women have been considered a delicate group during the novel pandemic, as little information has been available, especially early in the pandemic.

Data on maternal and neonatal outcomes of pregnant women infected with SARS-CoV-2 are scarce, mainly limited to case reports, case series or reviews. 
Clinical management guidelines are continuously evolving and any piece of information is valuable. However, as publications are coming from all over the world as soon as possible, it is understandable that the data is not systematised, thus making it difficult to interpret and questioning the reliability.

\section{OBJECTIVE}

We conducted a systematic review of available published papers on SARS-CoV-2 outcomes in pregnancy, with focus on neonatal effects and vertical transmission.

\section{METHODS}

\section{Search strategy and study selection}

We conducted a systematic literature research using Pubmed and Google Scholar covering the period from December 2019 to $20^{\text {th }}$ of November 2020. We used the following terms for search: COVID-19, pregnancy, congenital, perinatal, neonates/neonatal, vertical transmission. The titles and abstracts were reviewed by the authors in order to determine their relevance. Inclusion criteria were: articles written in English, laboratory confirmed COVID-19 infection in either pregnant woman or neonate with information regarding neonatal and maternal outcomes. Quality assessment on the included articles was not performed in order not to limit the data available even too scarce in the literature. Exclusion criteria included: cases not confirmed COVID-19, unreported maternal or neonatal outcomes. The systematic reviews found in the literature have already included the case reports and case series available, therefore we did not analyse individually each case series or case reports, but pooled all the reviews data. In regard to vertical transmission, we gathered all reports available and analysed each one.

\section{Data extraction and synthesis}

The review was conducted in accordance with PRISMA guidelines. The literature search returned 474 papers which were screened by title and abstract initially followed by retrieving full text where appropriate. Duplicates were identified through an independent manual screening. As the studies are very heterogenous, statistical analysis was not attempted, but the authors chose a narrative approach for reliable report of data available.
Relevant clinical information about neonates and pregnant women was extracted from studies included and was summarized in separate tables. The outcomes of pregnancies in women SARS-CoV-2 positive were pulled. The mother was considered to have COVID-19 infection if the RT-PCR from nasopharyngeal swab was positive. Neonate was considered to have COVID-19 infection if the RT-PCR from nasopharyngeal/oropharyngeal swab of the infant, blood/umbilical cord or amniotic fluid or placental biopsy was positive.

\section{RESULTS}

\section{Rates of COVID-19 in neonates of COVID-19 positive women}

We included 16 studies - systematic reviews and meta-analyses published between May 2020 and November 2020 which focused on perinatal outcomes of pregnant women with COVID-19. The number of studies analysed by each of the systematic reviews that we included ranged from 11 to 77 . The number of pregnant women included in the studies was between 68 and 11,432, with a total number of 27,546 (two studies did not report the number of pregnant women included, as the aim was to determine neonatal outcome, not maternal outcome).

The total number of neonates tested for COVID-19 was 5,906; according to each study analysed, it ranged between 46 and 1,992. The total number of COVID-19 positive neonates was 209. Overall, the rate of COVID-19 cases in neonates of COVID-19 positive mothers was $3 \%$ with $95 \%$ CI $[1.86,4.24]$ ); this was reported by 15 studies, and rates were ranging from 0 to $7 \%$. Data is summarised in Table 1.

\section{Outcomes of pregnancy in COVID-19 positive women}

Data on gestational age at birth was reported by 9 of the 16 studies included. Overall preterm birth rate was $16.4 \%$ with $95 \%$ CI $[10.5,22.3]$.

In pregnant women who tested positive for COVID-19, the overall rate of stillbirths and foetal deaths was $1.4 \%$ (11 studies, 0 to $4.8 \%$ ).

The overall rate of neonatal deaths was $0.5 \%(12$ studies, 0 to $2 \%$ ).

Data on maternal deaths was only reported by 2 studies - rate $0.4 \%$ and $4.1 \%$.

All informations are detailed in Table 1. 


\begin{tabular}{|c|c|c|c|c|c|c|c|c|c|c|c|c|}
\hline 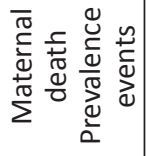 & $\begin{array}{l}\frac{\pi}{0} \\
\frac{\pi}{0} \\
0 \\
0 \\
0\end{array}$ & 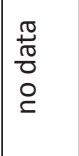 & \begin{tabular}{|l}
$\frac{\pi}{3}$ \\
$\frac{\pi}{0}$ \\
0 \\
0
\end{tabular} & $\begin{array}{l}\frac{\pi}{3} \\
\frac{\pi}{0} \\
\circ \\
\end{array}$ & $\begin{array}{l}\frac{\pi}{3} \\
\frac{\pi}{0} \\
0 \\
0\end{array}$ & \begin{tabular}{|l}
$\frac{\pi}{3}$ \\
$\frac{\pi}{0}$ \\
0 \\
0
\end{tabular} & $\begin{array}{l}\frac{\pi}{3} \\
\frac{\pi}{0} \\
0 \\
0\end{array}$ & $\begin{array}{l}\frac{\pi}{\pi} \\
\frac{\pi}{0} \\
0 \\
\end{array}$ & $\begin{array}{l}\frac{\pi}{3} \\
\frac{\pi}{0} \\
0 \\
0\end{array}$ & $\begin{array}{l}\frac{\pi}{3} \\
\frac{\pi}{0} \\
0 \\
0\end{array}$ & $\begin{array}{l}\frac{\pi}{3} \\
\frac{\pi}{0} \\
0 \\
0\end{array}$ & 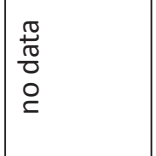 \\
\hline 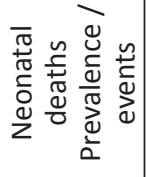 & 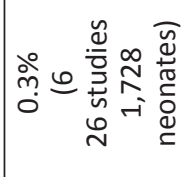 & $\begin{array}{l}\stackrel{0}{0} \\
\frac{\pi}{0} \\
0 \\
0 \\
1\end{array}$ & $\begin{array}{l}\bar{\Xi} \\
\circ \\
\partial \\
0 \\
0\end{array}$ & 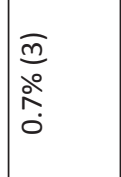 & \begin{tabular}{|l}
$\frac{\pi}{2}$ \\
$\frac{\pi}{0}$ \\
0 \\
0 \\
\multicolumn{1}{c}{}
\end{tabular} & 0 & 0 & 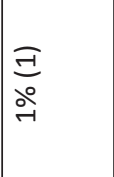 & $\begin{array}{l}\bar{\Xi} \\
\circ 0 \\
\partial \\
0\end{array}$ & $\begin{array}{l}\bar{\Xi} \\
\infty \\
\dot{0} \\
0 \\
0\end{array}$ & 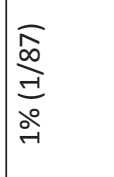 & $\begin{array}{l}\frac{\sigma}{0} \\
\dot{0} \\
\dot{0}\end{array}$ \\
\hline 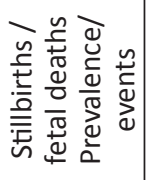 & 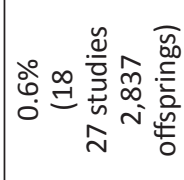 & $\begin{array}{l}\frac{\pi}{0} \\
\frac{\pi}{0} \\
0 \\
0\end{array}$ & $\begin{array}{l}\bar{\Xi} \\
\dot{0} \\
\dot{\sigma} \\
0\end{array}$ & $\begin{array}{l}\bar{a} \\
\vdots 0 \\
01 \\
0\end{array}$ & $\begin{array}{l}\frac{\pi}{0} \\
\frac{\pi}{0} \\
0 \\
\end{array}$ & $\begin{array}{l}\frac{\pi}{0} \\
\frac{\pi}{0} \\
0 \\
0\end{array}$ & 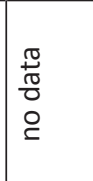 & $\begin{array}{l}\frac{\pi}{n} \\
\infty \\
0 \\
\dot{\sigma} \\
\dot{\sigma}\end{array}$ & 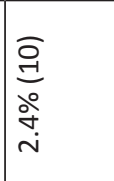 & 0 & 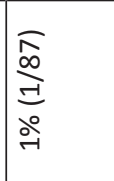 & 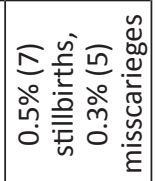 \\
\hline 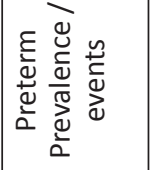 & 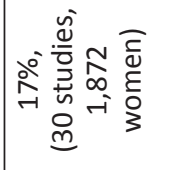 & $\begin{array}{l}\frac{\pi}{2} \\
\frac{\pi}{0} \\
0 \\
0\end{array}$ & $\begin{array}{l}\frac{\pi}{3} \\
\frac{\pi}{0} \\
0 \\
0 \\
1\end{array}$ & 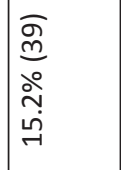 & $\begin{array}{l}\frac{\pi}{3} \\
\frac{\pi}{0} \\
0 \\
0 \\
0\end{array}$ & 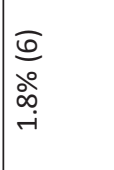 & 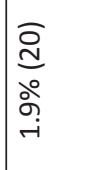 & 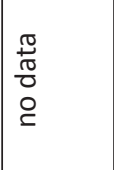 & 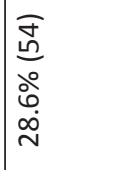 & 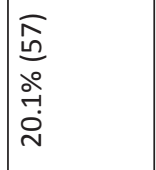 & $\begin{array}{l}\frac{\pi}{3} \\
\frac{\pi}{0} \\
0 \\
0\end{array}$ & \begin{tabular}{|l}
$\frac{\pi}{3}$ \\
$\frac{\pi}{0}$ \\
0 \\
0
\end{tabular} \\
\hline 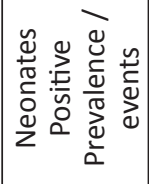 & $\begin{array}{l}0 \\
\mathbb{3} \\
\frac{\pi}{0} \\
0 \\
0\end{array}$ & $\stackrel{\circ}{\stackrel{+}{\circ} \widehat{\infty}}$ & 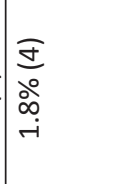 & 吾 & $\frac{\sqrt{n}}{2}$ & $\begin{array}{l}\stackrel{\bar{m}}{\stackrel{d}{n}} \\
\stackrel{0}{n}\end{array}$ & 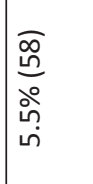 & 0 & $\begin{array}{l}\frac{\tilde{n}}{0} \\
\stackrel{\circ}{\dot{\sigma}} \\
-i\end{array}$ & 0 & $\underset{\ominus}{\vec{I}}$ & 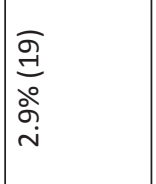 \\
\hline 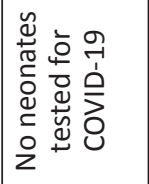 & $\begin{array}{l}\frac{\pi}{0} \\
\frac{\pi}{0} \\
0 \\
0\end{array}$ & $\stackrel{\Omega}{\neg}$ & $\vec{\sim}$ & 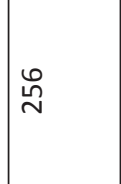 & $\vec{\lambda}$ & $\underset{m}{\stackrel{m}{m}}$ & \begin{tabular}{l}
$\infty$ \\
\multirow{2}{*}{} \\
$-i$
\end{tabular} & to & $\stackrel{\infty}{m}_{m}^{\infty}$ & 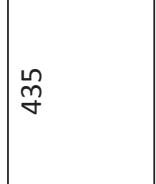 & $\stackrel{\text { Ln }}{\wedge}$ & 㞫 \\
\hline 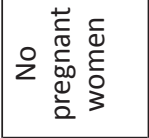 & $\begin{array}{l}\tilde{m} \\
\stackrel{-}{-} \\
\stackrel{-}{F}\end{array}$ & $\begin{array}{l}\frac{\pi}{0} \\
\frac{\pi}{0} \\
\circ \\
0\end{array}$ & $\begin{array}{l}\frac{\pi}{3} \\
\frac{\pi}{0} \\
0 \\
0\end{array}$ & $\stackrel{\substack{\infty \\
m}}{m}$ & $\infty$ & $\underset{m}{\infty}$ & $\begin{array}{l}\text { જે } \\
\text { م્ }\end{array}$ & $\underset{\sim}{ \pm}$ & $\begin{array}{l}\tilde{N} \\
\tilde{o} \\
\sigma\end{array}$ & 番 & $\underset{\sim}{\infty}$ & 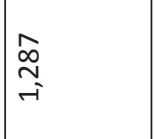 \\
\hline 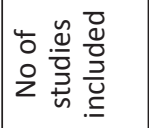 & $\curvearrowright$ & $\stackrel{m}{\rightarrow}$ & $\grave{N}$ & $\stackrel{m}{m}$ & $\stackrel{\sim}{\sim}$ & gి & 年 & $\stackrel{-}{-1}$ & $\stackrel{-}{-}$ & $\stackrel{m}{\rightarrow}$ & $\stackrel{\infty}{\rightarrow}$ & 8 \\
\hline$\stackrel{0}{\stackrel{\Perp}{*}}$ & 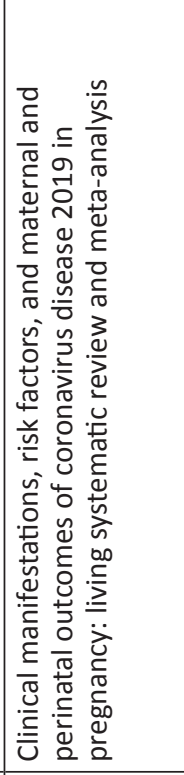 & 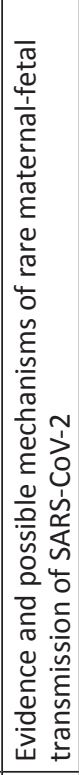 & 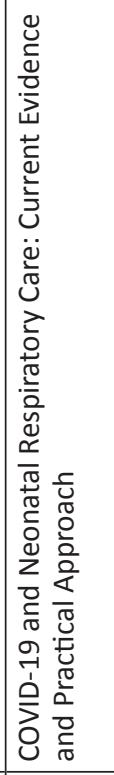 & 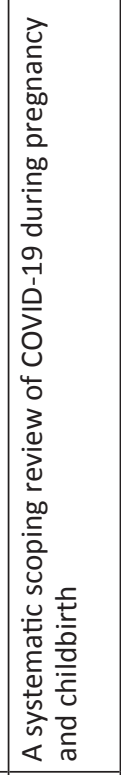 & 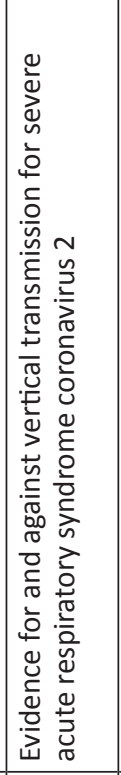 & 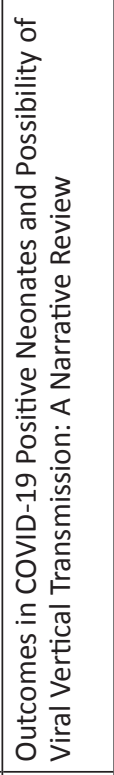 & 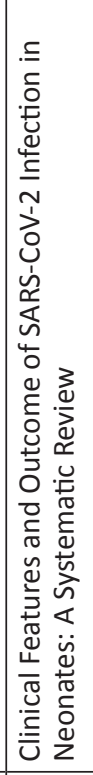 & 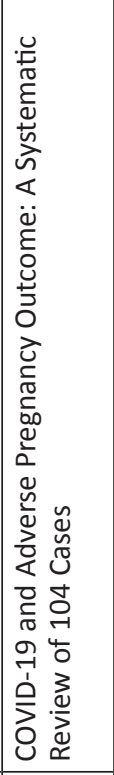 & 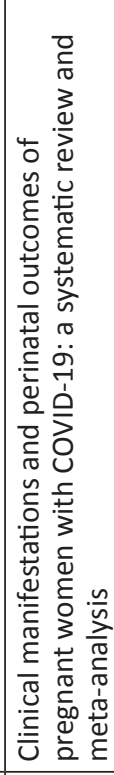 & 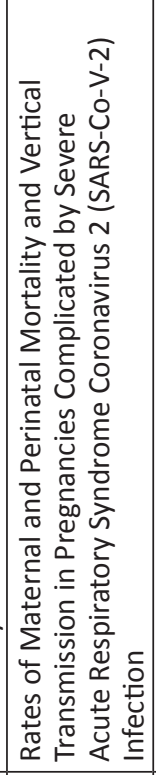 & 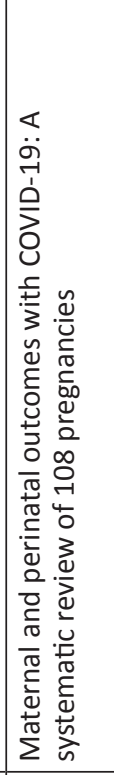 & 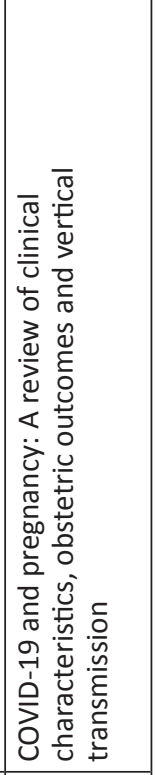 \\
\hline 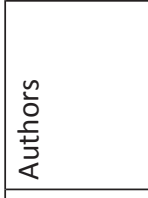 & 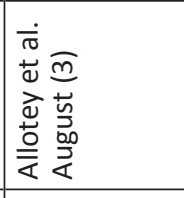 & 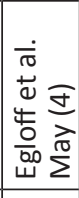 & 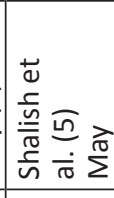 & 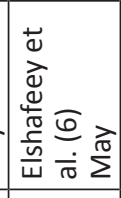 & 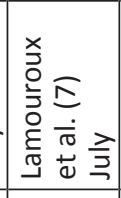 & 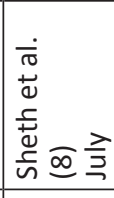 & 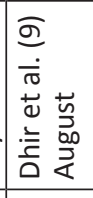 & 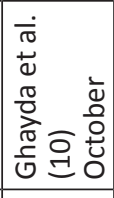 & 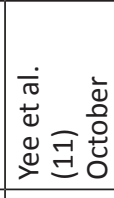 & 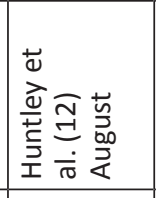 & 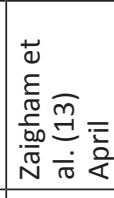 & 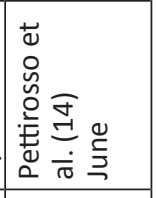 \\
\hline & $r$ & $\sim$ & $m$ & $\nabla$ & in & 6 & $\lambda$ & $\infty$ & $\sigma$ & 우 & $\exists$ & $\stackrel{\sim}{\sim}$ \\
\hline
\end{tabular}




\begin{tabular}{|c|c|c|c|c|}
\hline 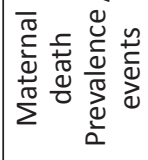 & 啇 & $\begin{array}{l}\frac{\pi}{0} \\
\frac{\pi}{0} \\
\circ \\
0\end{array}$ & $\begin{array}{l}\frac{\pi}{2} \\
\frac{\pi}{0} \\
0 \\
0\end{array}$ & 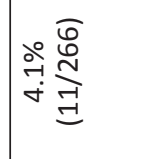 \\
\hline 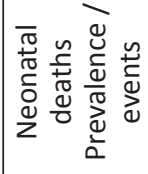 & $\begin{array}{l}\bar{m} \\
\stackrel{0}{\circ} \\
\grave{o}\end{array}$ & 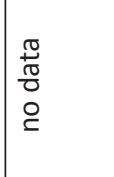 & $\begin{array}{l}\frac{\pi}{3} \\
\frac{\pi}{0} \\
0 \\
1\end{array}$ & 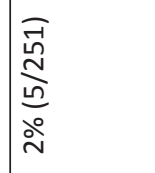 \\
\hline 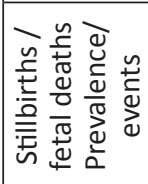 & 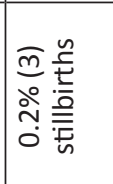 & $\begin{array}{l}\frac{\pi}{2} \\
\frac{\pi}{0} \\
0 \\
1\end{array}$ & 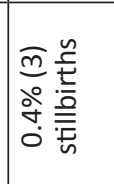 & 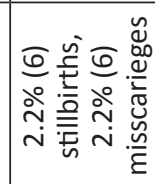 \\
\hline 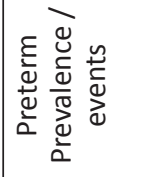 & $\stackrel{\stackrel{一}{\sim}}{\sim}$ & \begin{tabular}{|l}
$\frac{\pi}{2}$ \\
$\frac{\pi}{0}$ \\
0 \\
0 \\
\multicolumn{1}{c}{}
\end{tabular} & 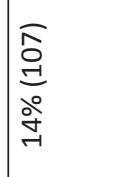 & 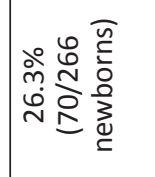 \\
\hline 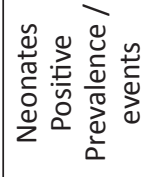 & 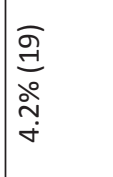 & 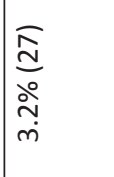 & $\begin{array}{l}\frac{\pi}{n} \\
\stackrel{0}{\circ} \\
\text { nn } \\
\text { nn }\end{array}$ & 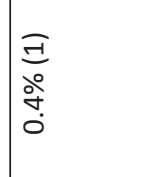 \\
\hline 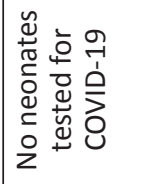 & 守 & $\begin{array}{l}0 \\
\tilde{n}\end{array}$ & ฉ్రి & 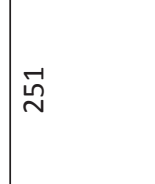 \\
\hline 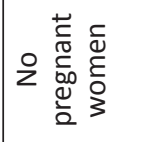 & $\underset{-}{\stackrel{\text { }}{-}}$ & \begin{tabular}{|l}
$\frac{\pi}{3}$ \\
$\frac{\pi}{0}$ \\
0 \\
0 \\
\end{tabular} & $\stackrel{\infty}{\stackrel{\infty}{\sim}}$ & $\begin{array}{l}\infty \\
\infty \\
m\end{array}$ \\
\hline 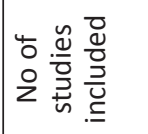 & $\stackrel{\searrow}{\sim}$ & ด & $\begin{array}{l}\frac{\pi}{2} \\
\frac{\pi}{0} \\
0 \\
9\end{array}$ & 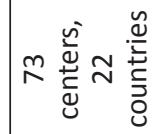 \\
\hline$\stackrel{0}{\stackrel{ \pm}{E}}$ & 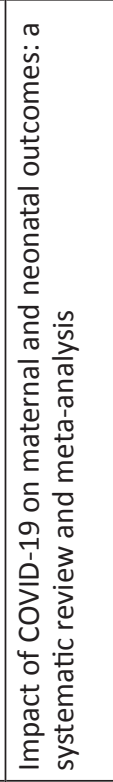 & 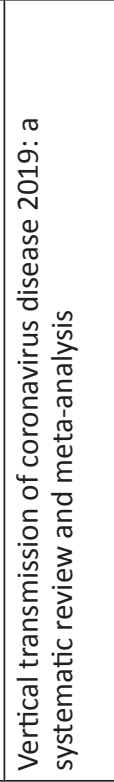 & 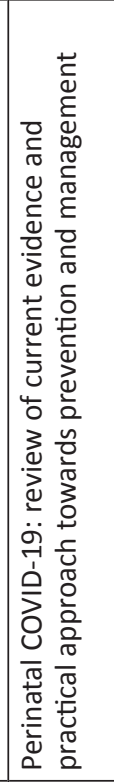 & 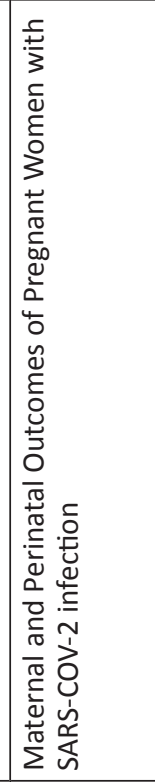 \\
\hline \multirow[t]{2}{*}{$\begin{array}{l}\frac{n}{0} \\
\frac{1}{5} \\
\frac{1}{2}\end{array}$} & 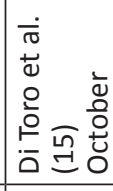 & 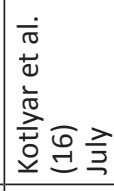 & 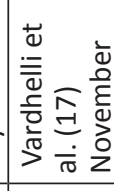 & 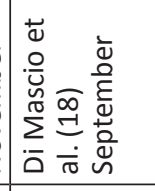 \\
\hline & $\stackrel{m}{\sim}$ & $\underset{\sim}{\sim}$ & $\stackrel{\Perp}{\sim}$ & $\underset{-1}{\varphi}$ \\
\hline
\end{tabular}

\section{Characteristics of neonates with vertically transmitted COVID-19}

We included in our review 7 case reports of neonates with vertical transmission of COVID-19. In total, 7 neonates with evidence of transplacental transmission were reported between March and September 2020 in: China, Iran, Canada, France, Germany, Saudi Arabia, USA. The sex ratio is 5:2 female to male newborns. The majority of newborns $n=5 / 7$ were born by caesarean section. Preterm birth occurred in $n=6 / 7$ cases, at a gestational age of 32 weeks to 35 weeks, with a median of 34 weeks. Birth weight was appropriate for gestational age for all newborns, ranging between $2,350 \mathrm{~g}$ and $3,280 \mathrm{~g}$. APGAR score was on the highest side for six newborns, respectively between 7 and 9 at 1 minute and 8 and 10 at 5 minutes. One baby received an APGAR score of 4 at 1 minute followed by 2 at 5 minutes. Majority of newborns ( $n=6$ out of 7) were admitted toneonatal intensive care unit (NICU) shortly after birth, respiratory support $(\mathrm{n}=1)$, neonatal resuscitation $(\mathrm{n}=1)$, encephalitic symptoms $(\mathrm{n}=1)$, hypoglycaemic episodes $(n=1)$, management of prematurity $(n=2)$. All these informations are presented in Table 2.

The symptoms of neonates with transplacental COVID-19 transmission are quite heterogenous. Three newborns had none/mild symptoms as follows: asymptomatic presentation $(\mathrm{n}=1)$, fever as only symptom $(\mathrm{n}=1)$ and fever plus minor respiratory symptoms $(\mathrm{n}=1)$. Four newborns had a severe presentation: one had severe respiratory distress with persistent pulmonary hypertension, one had persistent hypoglycaemia, hypothermia and feeding difficulties and two developed neurologic symptoms - in one case encephalitic signs started at 24 hours of life followed by respiratory distress at 80 hours of life while in the other case reported, neurologic signs (irritability, poor feeding, axial hypertonia, opisthotonus) started at 72 hours of life and MRI showed bilateral gliosis of the deep white periventricular and subcortical matter.

In regard to diagnosing vertical transmission of COVID-19, four of the cases had PCR positive for COVID-19 from amniotic fluid, placenta and nasopharyngeal swab at 24 hours, one case had placental swab and nasopharyngeal swab positive; in one case nasopharyngeal and rectal swab were positive. One case had repeated nasopharyngeal aspirate negative but positive IgM COVID-19 at 2 hours of life and 


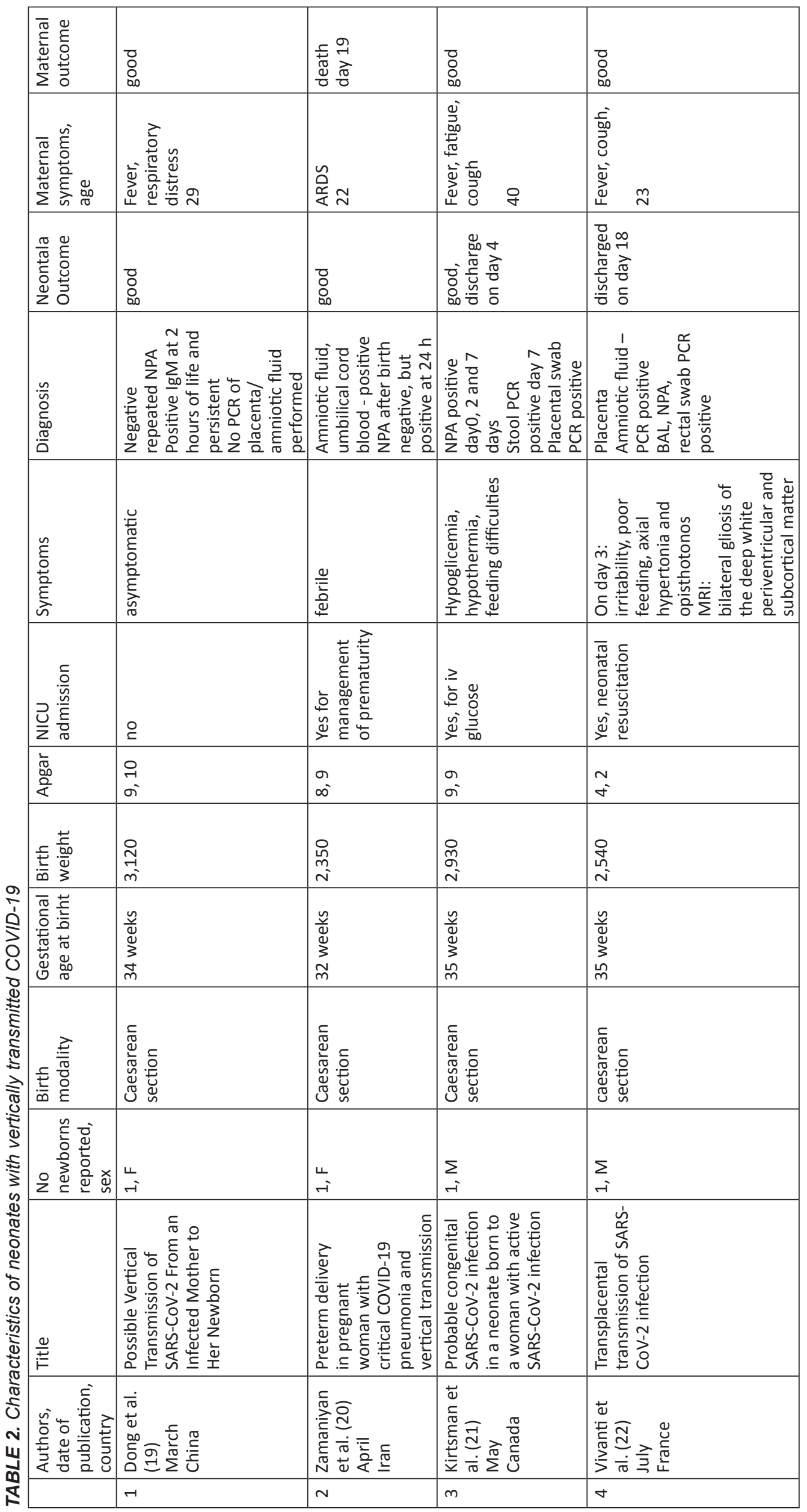




\begin{tabular}{|c|c|c|c|}
\hline 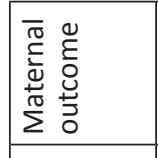 & 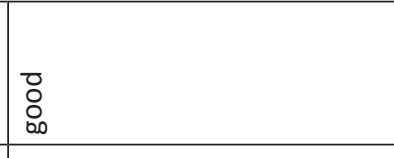 & \begin{tabular}{|l} 
\\
o \\
o.
\end{tabular} & \begin{tabular}{|l} 
\\
o \\
o.
\end{tabular} \\
\hline 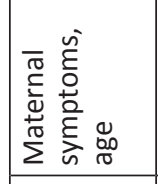 & 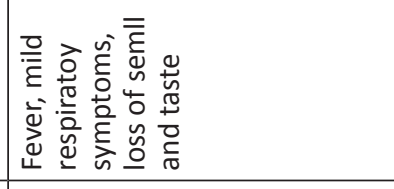 & 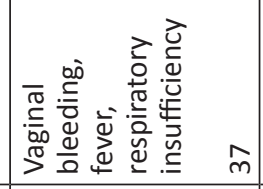 & 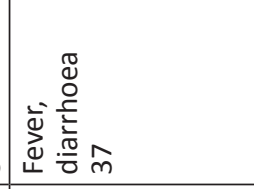 \\
\hline 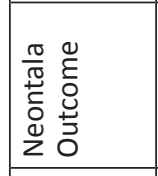 & 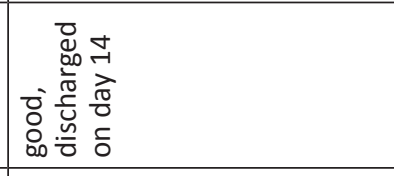 & 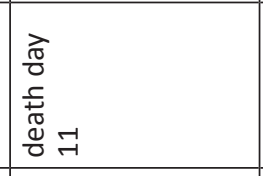 & 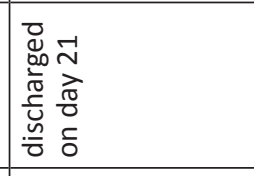 \\
\hline 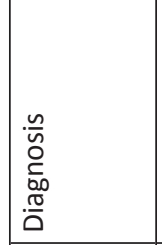 & 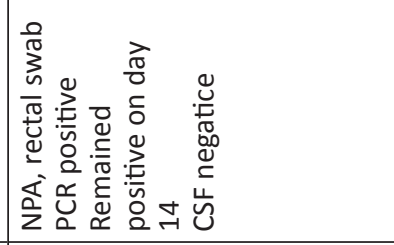 & 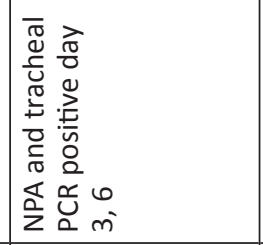 & 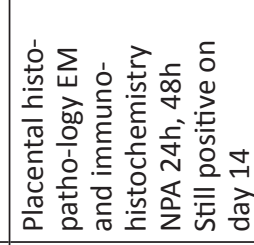 \\
\hline $\begin{array}{l}\text { है } \\
\text { है } \\
\text { है } \\
\text { s. }\end{array}$ & 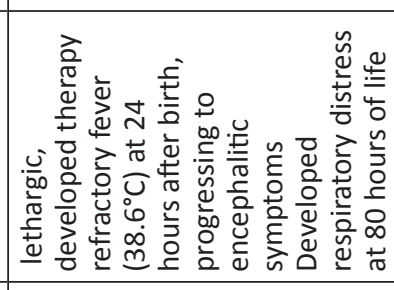 & 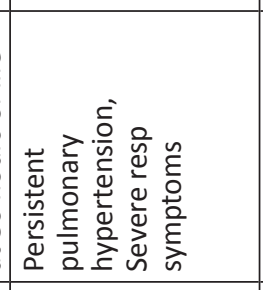 & 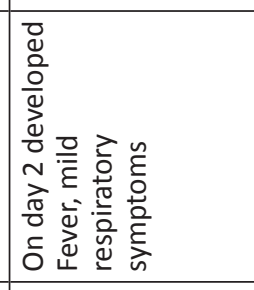 \\
\hline 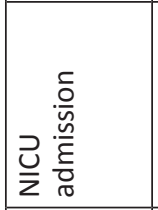 & 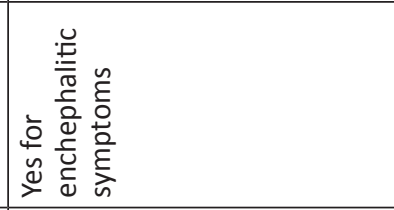 & 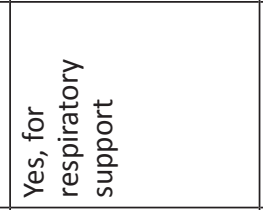 & 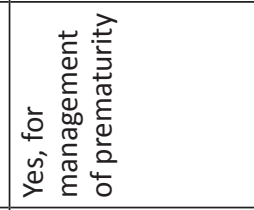 \\
\hline 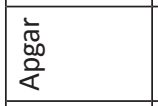 & $\begin{array}{l}a \\
\sigma \\
\sigma\end{array}$ & $\begin{array}{l}\infty \\
\infty \\
\infty\end{array}$ & I \\
\hline 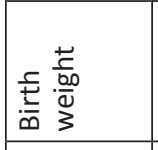 & 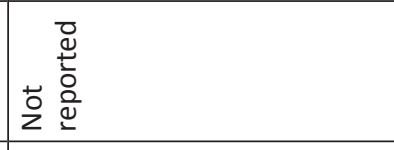 & 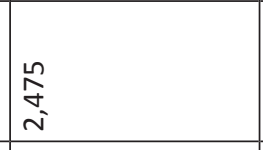 & $\mid$ \\
\hline 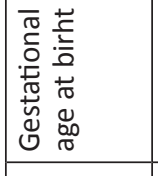 & 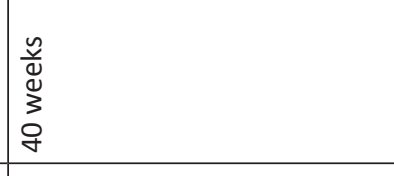 & 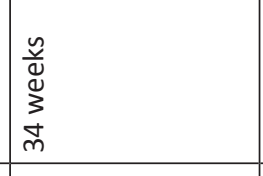 & 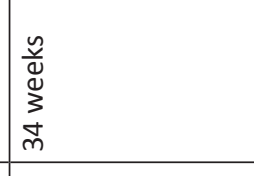 \\
\hline 辛 & 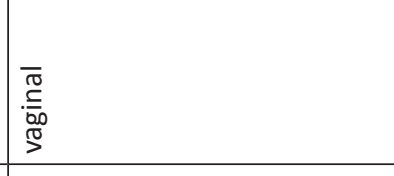 & 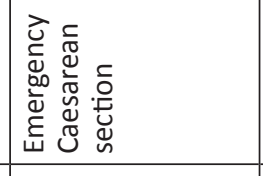 & 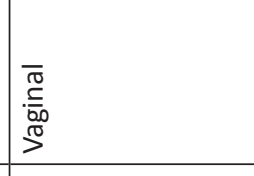 \\
\hline 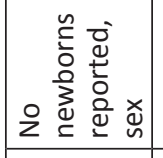 & 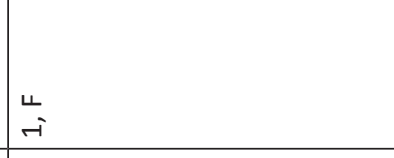 & $\mid \begin{array}{l}4 \\
-i \\
-1\end{array}$ & $\stackrel{u}{4}_{i}^{-1}$ \\
\hline 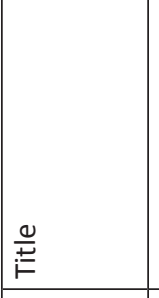 & 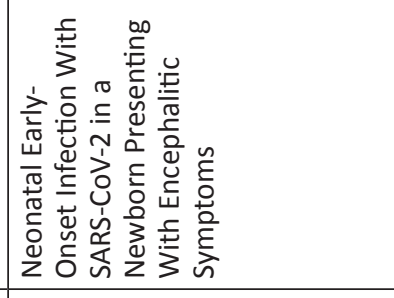 & 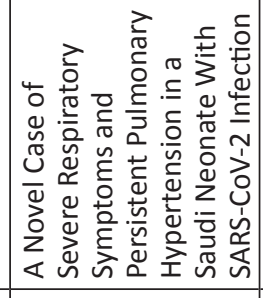 & 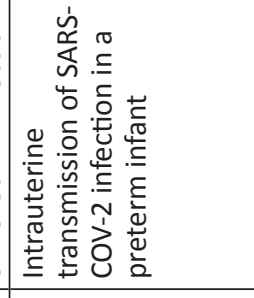 \\
\hline 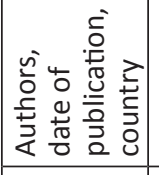 & 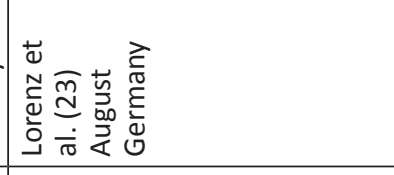 & 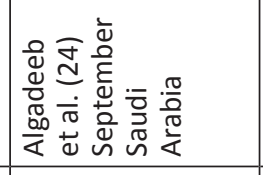 & 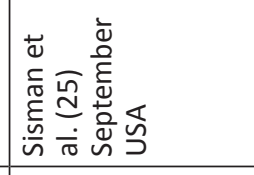 \\
\hline & ๓ & & \\
\hline
\end{tabular}


persistent; PCR of placenta/amniotic fluid were not performed.

Neonatal outcome was good in majority of cases, six newborns being discharged home in less than one month. One newborn who suffered from respiratory distress syndrome, cardiorespiratory failure, persistent pulmonary hypertension died on day 11 .

Analysing the characteristics of the mothers of the cases reported, we found that the age range was 22 years to 40 years, with three women aged $<30$ years old, three women aged $>35$ years old and in one case the age was not reported. Four mothers experienced minor symptoms: fever, mild respiratory symptoms along with loss of smell and taste in one of the cases and diarrhoea in another case. Three of the pregnant women presented with respiratory distress and fever and among them, one case associated vaginal bleeding. Six mothers recovered and were discharged home with their babies. One of the women who presented with respiratory distress deteriorated and died on day 19.

\section{DISCUSSION}

Our comprehensive review of studies describing SARS-CoV-2 infection in pregnancy, maternal and neonatal outcomes had analysed a high number of pregnant women COVID-19 positive and showed that the incidence of vertical transmission of COVID-19 was $3 \%$.

The outcomes were similar to general population. Preterm rate in pregnant women with COVID-19 infection was $16.4 \%$, comparable with the global incidence of $5-18 \%$ in the general pregnant population (26).

The rate of stillbirths was $1.4 \%$, lower to the one in general population $-1.8 \%$; although, majority of stillbirths are reported in low-income countries, while the studies included our review have come mainly from developed countries (26). However, these rates should be validated in bigger samples, and it is arguable that insufficient information about the causes of preterm or stillbirth is provided in order to draw definite conclusions. General worldwide screening and matched control studies should be performed to strengthen the findings (27).

Analysing the case reports of proved vertical transmission of SARS-CoV-2 virus, we found that although majority of neonates were born preterm, they were late preterm ( $>34$ weeks GA) with good
APGAR score and weight at birth. The symptoms were very heterogenous, with three newborns having mild symptoms (fever/minor respiratory symptoms) and four newborns developing severe symptoms. Interestingly, only one case exhibited severe respiratory picture with pneumonia and pulmonary hypertension, which is the usual presentation for severe COVID-19 in adults. In a study by Zhu et al. (28), 6 out of 10 infants born to COVID-19 mothers experienced respiratory symptoms, although all their PCR test were negative. Two of the newborns presented neurologic symptoms with onset at more than 24 hours of life and imaging evidence of brain changes in one case - bilateral gliosis of the deep white periventricular and subcortical matter.

In regard to assessing the modalities of diagnosing in utero transmission, we noted that according to availability of tests in each centre the data is variable. Most of the infants described in this review demonstrated SARS-CoV-2 virus in placenta and amniotic fluid along with nasopharyngeal swabs from neonates. One of the studies reported histologic findings of cytoplasmic staining for the SARS-CoV-2 nucleocapsid protein by immunohistochemistry and demonstrated viral particles by electron microscopy in the syncyiotrophoblastic cells strongly suggesting in utero transmission (25). In this particular case report the pregnant woman experienced premature rupture of membranes and the baby was born by vaginal delivery. Therefore, transmission could have occurred due to ascending infection and primary involvement of maternal gastrointestinal tract or by hematogenous spread during viremia caused by initial infection. Shanes et al. analysed 16 placentas of COVID-19 women who gave birth and found an increased rate of maternal vascular malperfusion, most prominently decidual arteriopathy including atherosis and fibrinoid necrosis and mural hyperthrophy of membrane arterioles. These changes have been associated with oligohydramnios, fetal growth restriction, preterm birth and stillbirth. However, none of the placentas in Shanes et al. study were tested for SARS-CoV-2 viral RNA and all infants have had a PCR swab test negative (29).

Four out of seven cases in our review had placental PCR, amniotic fluid and NPA of newborn positive. One case report published early in March 2020 did not have PCR of placenta/amniotic fluid performed but IgM antibodies were positive at 2 hours of life and on multiple repeated samples (19). 
The evolution of newborns was good, 6 out of 7 recovered and were discharged in 3-21 days. One death was reported and it was likely caused by the severe respiratory symptoms associated with pulmonary hypertension. In this case, prematurity could have contributed to the respiratory distress; however, the contribution of SARS-CoV-2 to lung pathology through angiotensin-converting enzyme 2 receptors is a plausible option.

Interestingly, 4 out of 7 pregnant women experienced mild symptoms (fever, cough or diarrhoea) while the other 3 presented with respiratory distress, one of them associating vaginal bleeding. The progress and outcome was good in 6 out of 7 cases, mothers being discharged home. In one case - a 22 years old pregnant woman which presented with acute respiratory distress death was reported; this was attributed to severe respiratory disease requiring ventilation and also to late presentation to the hospital, on day 4 of symptoms. World Association of Perinatal Medicine (30) reported a death rate of $0.4 \%$ among pregnant women with COVID-19 while Allotey et al. (3) found that increasing maternal age, high body mass index and pre-existing comorbidities are risk factors for severe COVID-19 in pregnant women - conditions similar to risk for severe disease in general population.

Vertical transmission is estimated at 3\% and it can by hypothesised that this is compatible with recent data reporting that placental cells coexpressing ACE2 and TMPRSS2 proteins required for SARS-CoV-2 cell entry are rare (31).

However, multiple questions remain unanswered at this point, such as what is the relation between se- vere COVID-19 disease in pregnancy and vertical transmission, long term effects of COVID-19 in newborns.

\section{Strengths and limitations}

Firstly, our review addresses very important clinical and research questions while there are uncertainties in regard to maternal and neonatal outcome of pregnancy in COVID-19 positive women, and equally important if vertical transmission is possible and what is the outcome.

We acknowledge that at the moment the scientific and medical world are keen to learn more about effect of COVID-19 as soon as possible in order to prevent and prepare for severe consequences. In light of this, many papers papers published on fast track and some might duplicate the information or mis-interpret. Hence, our review is looking at summarising and analysing reliable data from quality studies, only laboratory confirmed cases in order to provide some reliable answers.

One limitation is that not all studies have assessed same variables, therefore some results will be based on limited numbers of reports.

\section{CONCLUSIONS}

COVID-19 impact on pregnancy outcome is similar to general population in regard to preterm rate and stillbirth rate. Vertical transmission is possible and it seems to occur in about $3 \%$ of cases. Overall maternal and perinatal outcome is favourable and clinical presentation of in utero transmission of SARS-CoV-2 in newborns is heterogenous.

\section{REFERENCES}

1. World Health Organization. Coronavirus disease (COVID-2019) situation reports. Available at: https://www.who.int/publications/m/ item/weekly-epidemiological-update - 17-november-2020.

2. Sedgh G, Singh S, Hussain R. Intended and unintended pregnancies worldwide in 2012 and recent trends. Stud Fam Plann. 2014;45(3):301-314

3. Allotey J, Stallings E, Bonet M, et al. Clinical manifestations, risk factors, and maternal and perinatal outcomes of coronavirus disease 2019 in pregnancy: living systematic review and meta-analysis. BMJ. 2020 Sep 1;370:m3320.

4. Egloff C, Vauloup-Fellous C, Picone O, et al. Evidence and possible mechanisms of rare maternal-fetal transmission of SARS-CoV-2. J Clin Virol. 2020 Jul;128:104447.

5. Shalish W, Lakshminrusimha S, Manzoni P, et al. COVID-19 and Neonatal Respiratory Care: Current Evidence and Practical Approach. Am J Perinatol. 2020 Jun;37(8):780-791.

6. Elshafeey $F$, Magdi R, Hindi $N$, et al. A systematic scoping review of COVID-19 during pregnancy and childbirth. Int J Gynaecol Obstet. 2020 Jul;150(1):47-52.

7. Lamouroux A, Attie-Bitach T, Martinovic $\mathrm{J}$ et al. Evidence for and against vertical transmission for severe acute respiratory syndrome coronavirus 2. Am J Obstet Gynecol. 2020 Jul;223(1):91.e1-91.e4.

8. Sheth S, Shah N, Bhandari V. Outcomes in COVID-19 Positive Neonates and Possibility of Viral Vertical Transmission: A Narrative Review. Am J Perinatol. 2020 Oct;37(12):1208-1216.

9. Dhir SK, Kumar J, Meena J, et al. Clinical Features and Outcome of SARS-CoV-2 Infection in Neonates: A Systematic Review. J Trop Pediatr. 2020 Aug 28:fmaa059.

10. Ghayda RA, Li H, Lee KH, et al. COVID-19 and Adverse Pregnancy Outcome: A Systematic Review of 104 Cases. J Clin Med. 2020 Oct 26;9(11):E3441.

11. Yee J, Kim W, Han JM, et al. Clinical manifestations and perinatal outcomes of pregnant women with COVID-19: a systematic review and meta-analysis. Sci Rep. 2020 Oct 22;10(1):18126.

12. Huntley BJF, Huntley ES, Di Mascio D, et al. Rates of Maternal and Perinatal Mortality and Vertical Transmission in Pregnancies Complicated by Severe Acute Respiratory Syndrome Coronavirus 2 (SARS-Co-V-2) Infection: A Systematic Review. Obstet Gynecol. 2020 Aug;136(2):303-312. 
13. Zaigham $M$, Andersson $\mathrm{O}$. Maternal and perinatal outcomes with COVID-19: A systematic review of 108 pregnancies. Acta Obstet Gynecol Scand. 2020 Jul;99(7):823-829.

14. Pettirosso E, Giles M, Cole S, et al. COVID-19 and pregnancy: A review of clinical characteristics, obstetric outcomes and vertical transmission. Aust N Z J Obstet Gynaecol. 2020 Oct;60(5):640-659.

15. Di Toro F, Gjoka M, Di Lorenzo G, et al. Impact of COVID-19 on maternal and neonatal outcomes: a systematic review and meta-analysis. Clin Microbiol Infect. 2020 Nov 1:S1198743X(20)30618-2.

16. Kotlyar AM, Grechukhina O, Chen A, et al. Vertical transmission of coronavirus disease 2019: a systematic review and meta-analysis. Am J Obstet Gynecol. 2020 Jul 31:S0002-9378(20)30823-1.

17. Vardhelli V, Pandita A, Pillai A, et al. Perinatal COVID-19: review of current evidence and practical approach towards prevention and management. Eur J Pediatr. 2020 Nov 12:1-23.

18. Di Mascio D, Khalil A, Saccone G, et al. Outcome of coronavirus spectrum infections (SARS, MERS, COVID-19) during pregnancy: a systematic review and meta-analysis. Am J Obstet Gynecol MFM. 2020 May;2(2):100107.

19. Dong L, Tian J, He S, et al. Possible Vertical Transmission of SARS-CoV-2 From an Infected Mother to Her Newborn. JAMA. 2020 May 12;323(18):1846-1848.

20. Zamaniyan M, Ebadi A, Aghajanpoor S, et al. Preterm delivery, maternal death, and vertical transmission in a pregnant woman with COVID-19 infection. Prenat Diagn. 2020;40(13):1759-1761.

21. Kirtsman M, Diambomba Y, Poutanen SM, et al. Probable congenital SARS-CoV-2 infection in a neonate born to a woman with active SARS-CoV-2 infection. CMAJ. 2020 Jun 15;192(24):E647-E650.

22. Vivanti AJ, Vauloup-Fellous C, Prevot S, et al. Transplacental transmission of SARS-CoV-2 infection. Nat Commun. 2020 Jul 14;11(1):3572.

Conflict of interest: none declared

Financial support: none declared
23. Lorenz N, Treptow A, Schmidt S, et al. Neonatal Early-Onset Infection With SARS-CoV-2 in a Newborn Presenting With Encephalitic Symptoms. Pediatr Infect Dis J. 2020 Aug;39(8):e212.

24. Algadeeb KB, AlMousa HH, AlKadhem SM, et al. A Novel Case of Severe Respiratory Symptoms and Persistent Pulmonary Hypertension in a Saudi Neonate With SARS-CoV-2 Infection. Cureus. 2020 Sep 15;12(9):e10472.

25. Sisman J, Jaleel MA, Moreno W, et al. Intrauterine Transmission of SARS-COV-2 Infection in a Preterm Infant. Pediatr Infect Dis J. 2020 Sep;39(9):e265-e267.

26. WHO.int [homepage on the internet]. Preterm birth. Geneva, WHO, 2018, [accessed 20 November 2020]. Available at:https://www.who. int/news-room/fact-sheets/detail/preterm-birth.

27. WHO.int [homepage on the internet]. Stillbirth birth. Geneva, WHO, 2018, [accessed 20 November 2020]. Available at: https://www.who. int/reproductivehealth/topics/maternal_perinatal/stillbirth/en/.

28. Zhu H, Wang L, Fang C, et al. Clinical analysis of 10 neonates born to mothers with 2019-nCoV pneumonia. Transl Pediatr. 2020;9(1):51-60.

29. Shanes DE, Mithal LB, Otero $S$ et al. Placental pathology in COVID-19. Am J Clin Pathol. 2020 Jun;154(1):23-32.

30. WAPM (World Association of Perinatal Medicine) Working Group on COVID-19. Maternal and perinatal outcomes of pregnant women with SARS-CoV-2 infection. Ultrasound Obstet Gynecol. 2020 Sep 14.

31. Pique-Regi R, Romero R, Tarca A, et al. Does the human placenta express the canonical cell entry mediators for SARS-CoV-2? Elife 2020;9:e58716 